\title{
The Hirzebruch-Riemann-Roch Theorem
}

\author{
MADHAV V. NORI
}

\section{Dedicated to Professor William Fulton on his sixtieth birthday}

It is indeed an honor to dedicate this essentially self-contained proof of HRR to William Fulton, whose contributions to the study of Chow groups, intersection theory, and the Riemann-Roch theorems have led to a deeper understanding of these topics.

As is well known, Grothendieck formulated a relative version GRR of the Riemann-Roch for proper morphisms $f: X \rightarrow Y$, and HRR turned out to be the special case when $Y$ is a point. To prove GRR, Grothendieck showed that it sufficed to prove GRR for the projection $Y \times \mathbb{P}^{n} \rightarrow Y$ and for a closed immersion. The former is easy, but the latter is much more subtle; in parallel, Grothendieck also proved the Chern character induces an isomorphism

$$
\text { ch: } \mathbb{Q} \otimes K(X) \rightarrow \mathbb{Q} \otimes A(X) .
$$

Accounts of Grothendieck's method are found in [SGA; BS; M]. Fulton proved GRR without denominators for closed immersions quite directly by the famous "degeneration to the normal cone". This method has since been used in several related contexts (see e.g. $[\mathrm{Fa}]$ and $[\mathrm{GS}]$ ).

The aim of this note is to give a direct proof of HRR that does not rely of Grothendieck's method of factoring a morphism. What is crucially used here, however, is the formalism introduced by Grothendieck, and in particular the isomorphism $K(X) \rightarrow \mathcal{G}(X)$ of the $K$-groups of vector bundles and coherent sheaves, respectively, when $X$ is regular (the hypothesis of quasi-projectivity was removed by Kleiman; see $[\mathrm{F}]$ ). Our method can be extended to deduce GRR itself directly, but this has not been carried out here.

The HRR for compact complex manifolds was deduced by Atiyah and Singer from their index theorem; it was also proved by methods of differential geometry by Patodi [P] and Toledo-Tong [TT1]. What is more relevant to this paper is the Atiyah-Bott version of the Lefschetz fixed-point formula (see [AB]) adapted to cover the case where the set of fixed points is a submanifold. Such a version is due to Toledo and Tong (see [TT2]). The fixed-point formula we obtain in Section 2 for periodic self-maps is a little stronger than the classical formula when the characteristic of the ground field is positive: we get an identity in the Witt ring, which reduced modulo the characteristic yields the classical formula.

The Adams-Riemann-Roch is deduced from the fixed-point formula in Section 3. The Hirzebruch-Riemann-Roch is deduced from this in Section 4. The

Received March 13, 2000. Revision received March 17, 2000. 
reductions in Section 4 involve use of the Adams operations and are, for the most part, quite standard (see e.g. [GS]); thus, our exposition is brief.

If we take $n=2$ in Sections 2 and 3 then the paper would be about one-third in length, and this is enough to cover the Hirzebruch-Riemann-Roch whenever $\operatorname{char}(k) \neq 2$. Indeed, the paper rests on the following observation: If $\mathcal{F}$ is a coherent sheaf on a variety $X$, then the Lefschetz number of the natural involution $\sigma$ on the cohomologies of $\mathcal{F} \otimes \mathcal{F}$ on $X \times X$ is simply $\chi(X, \mathcal{F})$. The Riemann-Roch formalism is introduced in Section 1. The book by Fulton and Lang [FL] is a good reference for this section.

The author would like to thank Dipendra Prasad and S. Ramanan for useful discussions, and also Kaj Gartz for doing an excellent job of putting the manuscript into $\mathrm{T}_{\mathrm{E}} \mathrm{X}$.

\section{The Riemann-Roch Formalism}

We recall briefly the Adams power operations $\psi_{X}^{n}$ and the Bott classes $\theta^{n}(X)$, the Adams-Riemann-Roch, the Todd class, and the Hirzebruch-Riemann-Roch.

We use $K(X)$ to denote the Grothendieck group of locally free coherent sheaves of a scheme $X$, separated, and of finite type over $\operatorname{Spec}(k)$. For such a sheaf $\mathcal{F}$, its representative in $K(X)$ is denoted by $\operatorname{cl}(\mathcal{F})$. We often put $\delta(\mathcal{F})=$ $\sum_{p}(-1)^{p}\left(\bigwedge^{p} \mathcal{F}\right)$.

The formalism of Hirzebruch (see [FL]) associates to a power series $P \in$ $A[[t-1]]$ a homomorphism $A_{X}^{P}: K(X) \rightarrow A \otimes K(X)$ of Abelian groups, for every such scheme $X$ as just described, so that:

(a) $f^{*} \circ A_{Y}^{P}=A_{X}^{P} \circ f^{*}$ for every morphism $f: X \rightarrow Y$; and

(b) $A_{X}^{P}(\operatorname{cl}(\mathcal{L}))=P(\operatorname{cl}(\mathcal{L}))$ for every sheaf locally free of rank $1, \mathcal{L}$ on $X$.

Furthermore, the $A_{X}^{P}$ are uniquely determined by these properties.

If $P \in A[[t-1]]^{\times}$then there is a unique system of homomorphisms of Abelian groups, $M_{X}^{P}: K(X) \rightarrow(A \otimes K(X))^{\times}$, so that (a) and (b) hold.

Definition 1.1. $A=\mathbb{Z}, n \in \mathbb{Z}$, and $P(t)=t^{n}$. Then $A_{X}^{P}=\psi_{X}^{n}$ is the $n$th power operation of Adams.

For example: if $n=-1$, then $\psi_{X}^{n}(\operatorname{cl}(\mathcal{F}))=\operatorname{cl}\left(\mathcal{F}^{*}\right)$; if $n=2$, then $\psi_{X}^{n}(\operatorname{cl}(\mathcal{F}))=$ $\operatorname{cl}\left(\operatorname{Sym}^{2} \mathcal{F}\right)-\operatorname{cl}\left(\bigwedge^{2} \mathcal{F}\right)$.

Definition 1.2. $A=\mathbb{Z}\left[\frac{1}{n}\right], n$ is a nonzero integer, and $P(t)=\left(t^{n}-1\right) /(t-1)$. Then $\theta^{n}(X)=M_{X}^{P}\left(\operatorname{cl}\left(\Omega_{X}\right)\right)$ are Bott's cannibalistic classes, where $X$ is smooth.

With $n=-1$, for example, $\theta^{n}(X)=(-1)^{d} \operatorname{cl}\left(\Omega_{X}^{d}\right)$ where $d=\operatorname{dim}(X)$; for $n=$ 2, we have

$$
\theta^{n}(X)=\sum_{p} \operatorname{cl}\left(\Omega_{X}^{p}\right) .
$$

THEOREM (ARR). Assume that $X$ is smooth and complete. Then, for all $a \in$ $K(X)$, we have

$$
\chi(X, a)=\chi\left(X, \theta^{n}(X)^{-1} \cdot \psi_{X}^{n}(a)\right) .
$$


Definition 1.3. $R=\mathbb{Q}$ and $P(t)=\log (t) /(t-1)$. Then $\tilde{\operatorname{td}}(X)=M_{X}^{P}\left(\operatorname{cl}\left(\Omega_{X}\right)\right)$.

LEMMA 1.4. If $X$ is smooth of dimension $d$, then

$$
\theta^{n}(X) \cdot \psi_{X}^{n}(\tilde{\operatorname{td}}(X))=n^{\operatorname{dim}(X)} \tilde{\operatorname{td}}(X)
$$

Proof. Because $\psi_{X}^{n}$ is a ring homomorphism, we can see that $\psi_{X}^{n} \circ M_{X}^{P}=M_{X}^{Q}$ where $Q(t)=P\left(t^{n}\right)$ and $P \in \mathbb{Q}[[t-1]]^{\times}$. Thus, the lemma is a consequence of the identity

$$
\frac{t^{n}-1}{t-1} \cdot \frac{\log \left(t^{n}\right)}{t^{n}-1}=n\left(\frac{\log (t)}{t-1}\right) .
$$

In view of Lemma 1.4, ARR is equivalent to ARR'.

THEOREM (ARR'). If $X$ is smooth and complete of dimension $d$, and if $a \in$ $K(X)$, then

$$
\chi(X, a)=n^{-\operatorname{dim}(X)} \chi\left(\widetilde{\operatorname{td}}(X)^{-1} \cdot \psi_{X}^{n}(a \cdot \widetilde{\operatorname{td}}(X)) .\right.
$$

Definition-Notation 1.5. $A(X)=\bigoplus_{p} A^{p}(X)$ denotes the Chow ring, and ch: $\mathbb{Q} \otimes K(X) \rightarrow \mathbb{Q} \otimes A(X)$ denotes the Chern character. This is a ring homomorphism, and $\operatorname{ch}^{p} \circ \psi_{X}^{n}=n^{p} \cdot \psi_{X}^{n}$, where $\operatorname{ch}(a)=\sum_{p} \operatorname{ch}^{p}(a)$ and where $\operatorname{ch}^{p}(a) \in \mathbb{Q} \otimes A^{p}(X)$. Note that the Todd class of $X$, denoted by $\operatorname{td}(X)$, equals $\operatorname{ch}(\widetilde{\operatorname{td}}(X))$. For $\eta \in \mathbb{Q} \otimes A(X)$, write $\eta=\sum_{p} \eta^{p}$ with $\eta^{p} \in \mathbb{Q} \otimes A^{p}(X)$. Let $d=$ $\operatorname{dim} X$. The degree of the zero cycle $\eta^{d}$, denoted by $\operatorname{deg}\left(\eta^{d}\right)$, is written as $\int_{X} \eta$.

TheOREM (HRR). For all $\xi \in \mathbb{Q} \otimes K(X)$, where $X$ is smooth and complete, we have

$$
\chi(X, \xi)=\int_{X} \operatorname{ch}(\xi) \cdot \operatorname{td}(X) .
$$

That HRR implies ARR is obvious, simply because HRR gives a formula for $\chi(X, \xi)$ and for $\chi\left(X, \theta^{n}(X)^{-1} \cdot \psi_{X}^{n}(\xi)\right)$.

\section{The Atiyah-Bott-Lefschetz Formula}

We fix a perfect field $k$ and a natural number $n$ so that $k$ contains $n$ distinct $n$th roots of unity. The only schemes considered in this section are schemes $Y$ of finite type over $\operatorname{Spec}(k)$, equipped with an action of a cyclic group $G$ of order $n$. A generator $\sigma$ of $G$ will be fixed once and for all.

We will consider sheaves of $\mathcal{O}_{Y}$-modules on $Y$ equipped with $G$-action. The Grothendieck group of such sheaves that are locally free and finite rank (resp., coherent) will be denoted $K_{G}(Y)$ (resp., $\mathcal{G}_{G}(Y)$ ). Every subgroup $H$ of $G$ also acts on $Y$ and thus $K_{H}(Y)$ and $\mathcal{G}_{H}(Y)$ are also defined. For any $H$-sheaf $\mathcal{F}$ on $Y$ we have the induced representation $I_{H}^{G}(\mathcal{F})$, which is a $G$-sheaf on $Y$. This defines $I_{H}^{G}: K_{H}(Y) \rightarrow K_{G}(Y)$ and $I_{H}^{G}: \mathcal{G}_{H}(Y) \rightarrow \mathcal{G}_{G}(Y)$. If $H$ is generated by $\sigma^{a}$ with $a \mid n$, and if $\mathcal{F}$ is an $H$-sheaf on $Y$, note that $I_{H}^{G}(\mathcal{F})=\bigoplus_{i=0}^{a-1}\left(\sigma^{i}\right)^{*} \mathcal{F}$. If $\mathcal{A}$ is an $H$-sheaf on $Y$ and $\mathcal{B}$ is a $G$-sheaf on $Y$, then $\mathcal{B} \otimes I_{H}^{G}(\mathcal{A})=I_{H}^{G}(\mathcal{B} \otimes \mathcal{A})$. Thus we see that $I_{H}^{G}\left(K_{H}(Y)\right) \subset K_{G}(Y)$ is an ideal. We define $K_{\sigma}(Y)$ to be the quotient of 
$K_{G}(Y)$ by the sum of the $I_{H}^{G}\left(K_{H}(Y)\right)$ taken over all proper subgroups $H$ of $G$; we define $\mathcal{G}_{\sigma}(Y)$ in a like manner. Thus $K_{\sigma}(Y)$ is a ring, $\mathcal{G}_{\sigma}(Y)$ is a $K_{\sigma}(Y)$-module, and the natural arrow $K_{\sigma}(Y) \rightarrow \mathcal{G}_{\sigma}(Y)$ is a module homomorphism.

LEMMA 2.1. If $Y$ is a regular scheme, then

(a) $K_{G}(Y) \rightarrow \mathcal{G}_{G}(Y)$ and

(b) $K_{\sigma}(Y) \rightarrow \mathcal{G}_{\sigma}(Y)$

are both isomorphisms.

Proof. The standard method of proof (see e.g. [F, Apx. B.8.3]) shows that $K_{G}(Y) \rightarrow \mathcal{G}_{G}(Y)$ is an isomorphism, once it is checked that every coherent $G$-sheaf $\mathcal{F}$ on $Y$ is a quotient of a locally free $G$-sheaf on $Y$ of finite rank. An epimorphism $\mathcal{A} \rightarrow \mathcal{F}$ of ordinary sheaves induces a $G$-epimorphism $I_{H}^{G}(\mathcal{A}) \rightarrow \mathcal{F}$ where $H=\{e\}$. Taking $\mathcal{A}$ to be locally free of finite rank, the result follows.

Part (b) is a consequence of part (a) because $G$ can be replaced by any proper subgroup $H$ in part (a).

Lemma 2.2. Let $F$ be a $G$-stable closed subset of $Y$ and let $U$ be its complement. Let $i: F \rightarrow Y$ and $j: U \rightarrow Y$ denote the inclusions. Then $i_{*}$ and $j^{*}$ induce the exact sequences

(a) $\mathcal{G}_{G}(F) \rightarrow \mathcal{G}_{G}(Y) \rightarrow \mathcal{G}_{G}(U) \rightarrow 0$ and

(b) $\mathcal{G}_{\sigma}(F) \rightarrow \mathcal{G}_{\sigma}(Y) \rightarrow \mathcal{G}_{\sigma}(U) \rightarrow 0$.

Proof. For part (a), again the standard proof applies. Exactness at $\mathcal{G}_{G}(Y)$ is the issue, and for this we need $\phi: \mathcal{G}_{G}(U) \rightarrow T=\operatorname{coker}\left(i_{*}: \mathcal{G}_{G}(F) \rightarrow \mathcal{G}_{G}(Y)\right)$. For a coherent $G$-sheaf $\mathcal{F}$ on $U$, choose a coherent $G$-subsheaf $\mathcal{F}^{\prime}$ of $j_{*} \mathcal{F}$ so that $\mathcal{F}^{\prime} \mid U=\mathcal{F}$. We define $\phi(\operatorname{cl}(\mathcal{F}))=\operatorname{cl}\left(\mathcal{F}^{\prime}\right)$ in $T$.

For part (b), one need only note that (a) is valid for all proper subgroups $H$ of $G$ as well.

LEMMA 2.3. If $\sigma$ acts without fixed points on $Y$ (i.e., if $\{y \in Y(\bar{k}) \mid \sigma y=y\}=$ $\emptyset)$, then

$$
\mathbb{Z}\left[\frac{1}{n}\right] \otimes \mathcal{G}_{\sigma}(Y)=0 .
$$

Proof. We may choose a $G$-stable, nonempty Zariski-open affine subset $U$ of $Y$ such that the $G$-action of $U$ comes from a fixed-point-free action of $G / H$ where $H$ is a proper subgroup. We attempt to prove the lemma for $U$ first. If $\mathcal{F}$ is a $G$-sheaf on $U$, then $I_{H}^{G}(\mathcal{F})=\mathcal{F} \otimes I_{H}^{G}\left(\mathcal{O}_{U}\right)$. Thus, if $I_{H}^{G}\left(\mathcal{O}_{U}\right) \cong \mathcal{O}_{U}^{a}$ as a $G$-sheaf, where $a=\#(G / H)$, it would follow that $a \cdot \mathcal{G}_{G}(U)=0$. Let $\pi: U \rightarrow V$ be the quotient by the $(G / H)$-action. By descent, the $(G / H)$-sheaf $I_{H}^{G}\left(\mathcal{O}_{U}\right)=\pi^{*} \mathcal{A}$ for some locally free rank- $a$ sheaf $\mathcal{A}$ on $V$; actually $\mathcal{A}=\pi_{*} \mathcal{O}_{U}$, but this does not concern us. Replacing $V$ by a suitable nonempty open $V^{\prime}$ and replacing $U$ by $\pi^{-1}\left(V^{\prime}\right)$, we may assume that $\mathcal{A} \cong \mathcal{O}_{V}^{a}$. Induction on dimension and Lemma 2.2 now show that $\mathcal{G}_{\sigma}(Y)$ is annihilated by $n^{e}$, where $e=1+\operatorname{dim} Y$.

Proposition 2.4. Let FY denote the closed subscheme of fixed points of $\sigma$ in $Y$. Let $i: F Y \rightarrow Y$ denote the inclusion. Then $i_{*}: \mathbb{Z}\left[\frac{1}{n}\right] \otimes \mathcal{G}_{\sigma}(F Y) \rightarrow \mathbb{Z}\left[\frac{1}{n}\right] \otimes \mathcal{G}_{\sigma}(Y)$ is surjective. 
Proof. This is an immediate consequence of Lemmas 2.3 and 2.2.

REMARK 2.5. In Proposition 2.4, $i_{*}$ is an isomorphism, as we can see from Quillen's exact sequence (see [Q, Thm. 5.4, p. 131] adapted to $\tilde{\mathcal{G}}$ ). We prove this here only when $Y$ is smooth (see Theorem 2.8).

NOTATION 2.6. Let $R(k)$ be the subring generated by $\frac{1}{n}$ and all the $n$th roots on unity in $k$ (resp., the Witt ring $W(k)$ of $k$ ) if $\operatorname{char}(k)=0$ (resp., $>0$ ). We have a ring homomorphism $R(k) \rightarrow k$, and every $n$th root of unity $\lambda$ in $k$ lifts uniquely to an $n$th root of unity $\langle\lambda\rangle$ in $R(k)$.

Let $\hat{G}=\operatorname{Hom}\left(G, k^{\times}\right)$. Let $Y$ be a scheme on which $G$ acts trivially (this will be applied to $\operatorname{Spec}(k)$ and $F Y$ in the sequel). Every $G$-sheaf $\mathcal{F}$ on $Y$ is the direct sum of its eigensheaves $\mathcal{F}_{g}$ for $g \in \hat{G}$. Thus $\mathcal{G}_{G}(Y)=\mathbb{Z}[\hat{G}] \otimes \mathcal{G}(Y)$. For $\tau \in G$ we define

$$
\operatorname{tr}(\tau \mid \mathcal{F})=\sum_{g \in \hat{G}}\langle g(\tau)\rangle \operatorname{cl}\left(\mathcal{F}_{g}\right) .
$$

This extends to an $R(k)$-module homomorphism

$$
\operatorname{tr}(\tau \mid \cdot): R(k) \otimes \mathcal{G}_{G}(Y) \rightarrow R(k),
$$

which is an $R(k)$-algebra homomorphism if $Y$ is smooth.

Put gen $=\{\tau \in G \mid \tau$ generates $G\}$. Taking all $\tau \in G$ and then all $\tau \in$ gen, we obtain the isomorphisms

$$
\begin{aligned}
& \operatorname{tr}: R(k) \otimes \mathcal{G}_{G}(Y) \rightarrow \mathcal{G}(Y)^{G}, \\
& \operatorname{tr}: R(k) \otimes \mathcal{G}_{\sigma}(Y) \rightarrow \mathcal{G}(Y)^{\text {gen }} .
\end{aligned}
$$

These arrows are $R(k)$-algebra homomorphisms if $Y$ is smooth.

Notation 2.7. We put $\delta(\mathcal{F})=\sum_{p}(-1)^{p} \operatorname{cl}\left(\bigwedge^{p} \mathcal{F}\right)$ for a locally free sheaf $\mathcal{F}$.

THEOREM 2.8. If $Y$ is smooth, then so is FY. Let I be the sheaf of ideals in $\mathcal{O}_{Y}$ that vanish on $F Y$. Then:

(a) $\delta\left(I / I^{2}\right)$ is a unit in $\mathbb{Z}\left[\frac{1}{n}\right] \otimes \mathcal{G}_{\sigma}(F Y)$;

(b) $i_{*}: \mathbb{Z}\left[\frac{1}{n}\right] \otimes \mathcal{G}_{\sigma}(F Y) \rightarrow \mathbb{Z}\left[\frac{1}{n}\right] \otimes \mathcal{G}_{\sigma}(Y)$ is an isomorphism; and

(c) $i^{*} i_{*} a=a \delta\left(I / I^{2}\right)$ for all $a \in \mathbb{Z}\left[\frac{1}{n}\right] \otimes \mathcal{G}_{\sigma}(F Y)$.

Proof. If $\mathcal{F}$ is locally free on $F Y$, then $\operatorname{tor}_{p}^{\mathcal{O}_{Y}}\left(\mathcal{F}, \mathcal{O}_{F Y}\right) \cong \mathcal{F} \otimes \bigwedge^{p}\left(I / I^{2}\right)$, so this proves part (c). From Proposition 2.4, we see that (a) implies (b).

Because $I / I^{2}=\bigoplus_{g \in \hat{G}}\left(I / I^{2}\right)_{g}$ we see that $\delta\left(I / I^{2}\right)=\prod_{g \in \hat{G}} \delta\left(I / I^{2}\right)_{g}$. Because $R(k)$ is a nonzero free $\mathbb{Z}\left[\frac{1}{n}\right]$-module, it suffices to check that $\delta\left(I / I^{2}\right)_{g}$ is a unit in $R(k) \otimes \mathcal{G}_{\sigma}(F Y)$; in view of 2.6, this is the same as checking that $\operatorname{tr}\left(\tau \mid \delta\left(I / I^{2}\right)_{g}\right)$ is a unit in $R(k) \otimes \mathcal{G}(F Y)$ for every $\tau \in$ gen and every $g \in G$. Let $r(g)$ be the rank of $\left(I / I^{2}\right)_{g}$. Because $\{a \in K(F Y) \mid \operatorname{rank}(a)=0\}$ is a nilpotent ideal, it suffices to check that $\operatorname{rank} \operatorname{tr}\left(\tau \mid \delta\left(I / I^{2}\right)_{g}\right)=(1-\langle g(\tau)\rangle)^{r(g)}$ is a unit in $R(k)$.

Because $F Y$ is also the fixed points of $\tau$, no eigenvalue of $\tau$ on $I / I^{2}$ can equal 1 . Thus, if $r(g)$ is positive at some point of $Y$, then $g(\tau) \neq 1$. Thus $\langle g(\tau)\rangle \neq 1$, and that $1-\langle g(\tau)\rangle$ is a unit in $R(k)$ is standard. 
Notation 2.9. We will assume that $Y$ is complete. Then we have $\chi_{G}(Y, \cdot)$ : $\mathcal{G}_{G}(Y) \rightarrow K_{G}(\operatorname{Spec}(k))$ given by $\chi_{G}(Y, \operatorname{cl} \mathcal{F})=\sum_{p}(-1)^{p} \operatorname{cl}\left(H^{p}(Y, \mathcal{F})\right)$. Because $I_{H}^{G} H^{p}(Y, \mathcal{F})=H^{p}\left(Y, I_{H}^{G}(\mathcal{F})\right)$ for an $H$-sheaf $\mathcal{F}$ on $Y$, the $\chi_{G}(Y, \cdot)$ factors through as

$$
\chi_{\sigma}(Y, \cdot): \mathcal{G}_{\sigma}(Y) \rightarrow K_{\sigma}(\operatorname{Spec}(k))
$$

We put $\operatorname{Lef}(\sigma, Y, a)=\operatorname{tr}\left(\sigma \mid \chi_{\sigma}(Y, a)\right)$ with $\operatorname{tr}(\sigma \mid \cdot)$ as in 2.6. Clearly, if $G$ acts trivially on $Y$ then

$$
\operatorname{Lef}(\sigma, Y, a)=\chi(Y, \operatorname{tr}(\sigma \mid a)) .
$$

THeORem 2.10. Let $Y$ be smooth and complete. Let $a \in \mathbb{Z}\left[\frac{1}{n}\right] \otimes \mathcal{G}_{\sigma}(Y)$ and put $b=\delta\left(I / I^{2}\right)^{-1} \cdot i^{*} a$, with the notation of 2.7 ; thus $b \in \mathbb{Z}\left[\frac{1}{n}\right] \otimes \mathcal{G}_{\sigma}(F Y)$. Then $\operatorname{Lef}(\sigma, Y, a)=\chi(F Y, \operatorname{tr}(\sigma \mid b))$.

Proof. From Theorem 2.8, we see that $i_{*} b=a$. Because $\chi_{\sigma}\left(Y, i_{*} b\right)=\chi_{\sigma}(F Y, b)$, the result follows from the setup in 2.9 .

\section{The Adams-Riemann-Roch Theorem}

We now apply the results of the previous section to the following special situation. Let $X$ be a smooth variety defined over $k$, and let $Y=X^{n}$ be equipped with the natural action of the permutation group. We choose an $n$-cycle $\sigma$ once and for all and denote by $G$ the subgroup generated by $\sigma$. We denote by $i: X \rightarrow Y$ the diagonal embedding; in the notation of the previous section this is $F Y$, the fixed points of $\sigma$ in $Y$.

LEMMA 3.1. For a coherent sheaf $\mathcal{F}$ on $X$, we put $S \mathcal{F}=\mathcal{F} \otimes \mathcal{F} \otimes \cdots \otimes \mathcal{F}$ on $Y$. Thus $S \mathcal{F}$ is a $G$-sheaf. There is an additive homomorphism $S: \mathcal{G}(X) \rightarrow \mathcal{G}_{\sigma}(Y)$, so that $S(\operatorname{cl}(\mathcal{F}))=\operatorname{cl}(S \mathcal{F})$ for all coherent $\mathcal{F}$ on $X$.

Proof. Let $0 \rightarrow \mathcal{F}^{\prime} \rightarrow \mathcal{F} \rightarrow \mathcal{F}^{\prime \prime} \rightarrow 0$ be a short exact sequence of coherent sheaves on $X$. We put

$$
\mathcal{F}=F^{0} \mathcal{F} \supset F^{1} \mathcal{F}=\mathcal{F}^{\prime} \supset F^{2} \mathcal{F}=0 .
$$

The decreasing filtration $F^{\bullet} \mathcal{F}$ on $\mathcal{F}$ induces a decreasing filtration $F \cdot S \mathcal{F}$ of $S \mathcal{F}$ for which we see that $F^{0} S \mathcal{F}=S \mathcal{F}, F^{n+1} S \mathcal{F}=0$, and:

(1) $F^{0} S \mathcal{F} / F^{1} S \mathcal{F} \cong S \mathcal{F}^{\prime \prime}$ and $F^{n} S \mathcal{F} / F^{n+1} S \mathcal{F} \cong S \mathcal{F}^{\prime}$;

(2) for $0<p<n, F^{p} S \mathcal{F} / F^{p+1} S \mathcal{F}$ is a direct sum of sheaves induced from proper subgroups of $G$.

Thus, $\operatorname{cl}(S \mathcal{F})=\operatorname{cl}\left(S \mathcal{F}^{\prime}\right)+\operatorname{cl}\left(S \mathcal{F}^{\prime \prime}\right)$ in $\mathcal{G}_{\sigma}(Y)$, and the lemma follows.

Naturally, we also have $S: K(X) \rightarrow K_{\sigma}(Y)$.

LeMma 3.2. For all $a$ in $K(X)$, we have

$$
i^{*} S a=\psi_{X}^{n} a \text { in } \mathbb{Z}\left[\frac{1}{n}\right] \otimes K_{\sigma}(X) .
$$


LEMMA 3.3. If $X$ is smooth, then

$$
\delta\left(\frac{I}{I^{2}}\right)=\theta^{n}(X) \text { in } \mathbb{Z}\left[\frac{1}{n}\right] \otimes K_{\sigma}(X) .
$$

The right-hand sides of the equations in Lemmas 3.2 and 3.3 belong to $K(X)$, and we have a natural arrow $K(X) \rightarrow K_{\sigma}(X)$ because $G$ acts trivially on $X$. The Adams operations $\psi_{X}^{n}$ and the Bott classes $\theta^{n}(X)$ have been defined in 1.1 and 1.2, respectively, and $\delta\left(I / I^{2}\right)$ occurs in 2.7 and 2.8 .

Proof of Lemma 3.2. For every generator $\tau$ of $G$, the operation $a \mapsto \operatorname{tr}\left(\tau \mid i^{*} S a\right)$ is additive in $a$ by Lemma 3.1, and it is obviously functorial in $X$. If $a=$ $\operatorname{cl}(\mathcal{L})$ where $\mathcal{L}$ is locally free of rank 1 , then the $G$-action on $S \mathcal{L}$ is trivial and $\operatorname{cl}\left(i^{*} S \mathcal{L}\right)=a^{n}$. Since these properties characterize the Adams operations, we see that $\operatorname{tr}\left(\tau \mid i^{*} S a\right)=\psi_{X}^{n} a$ in $R(k) \otimes K(X)$. From 2.6, it follows that $i^{*} S a=\psi_{X}^{n} a$ in $R(k) \otimes K_{\sigma}(X)$, and this implies the result.

Proof of Lemma 3.3. We set $\bigwedge(\mathcal{A})=\sum_{p}(-T)^{p} \operatorname{cl}\left(\bigwedge^{p} \mathcal{A}\right)$ in $\mathbb{Z}[T] \otimes K_{\sigma}(X)$ for every locally free $G$-sheaf $\mathcal{A}$ on $X$. For every locally free sheaf $\mathcal{F}$ with trivial $G$-action, we have an exact sequence of $G$-sheaves on $X$ :

$$
0 \rightarrow s \mathcal{F} \rightarrow \mathcal{F}^{n} \rightarrow \mathcal{F} \rightarrow 0,
$$

where $\mathcal{F}^{n}=I_{H}^{G}(\mathcal{F})$ and $H=\{e\}$. If $\mathcal{F}=\Omega_{X}$, then $s \mathcal{F}=I / I^{2}$ and thus $\delta\left(I / I^{2}\right)$ is the value, at $T=1$, of $\bigwedge\left(s \Omega_{X}\right)$. Let $\tau$ be a generator of $G$ and put $L(\mathcal{F})=\operatorname{tr}(\tau \mid \bigwedge(s \mathcal{F}))$. We first compute $\operatorname{tr}\left(\tau \mid \bigwedge\left(\mathcal{L}^{n}\right)\right)$ where $\mathcal{L}$ is locally free of rank 1 on $X$. Because the action of $\tau$ on $\{S \subset\{1,2, \ldots, n\} \mid \# S=p\}$, where $0<p<n$, is fixed-point-free, it follows that $\operatorname{cl}\left(\wedge^{p} \mathcal{L}^{n}\right)=0$ in this range. Putting $a=\operatorname{cl}(\mathcal{L})$, we see that $\operatorname{tr}\left(\tau \mid \bigwedge\left(\mathcal{L}^{n}\right)\right)=1+\langle\varepsilon(\tau)\rangle a^{n}(-T)^{n}=$ $1-(a T)^{n}$, where $\varepsilon$ denotes the sign of the permutation. Also, $\operatorname{tr}(\tau \mid \wedge(\mathcal{L}))=$ $1-a T$ and $\operatorname{tr}(\tau \mid \bigwedge(\mathcal{L})) \cdot \operatorname{tr}(\tau \mid \bigwedge(s \mathcal{L}))=\operatorname{tr}\left(\tau \mid \bigwedge\left(\mathcal{L}^{n}\right)\right)$. Furthermore, $1-a T$ is not a zero divisor in $R(k)[T] \otimes K(X)$ because its rank, $1-T$, is not a zero divisor in $R(k)[T]$. Setting $P(t)=\left(t^{n}-1\right) /(t-1)$, we see that $\operatorname{tr}(\tau \mid \bigwedge(s \mathcal{L}))=$ $P(a T)$ and, evaluating at $T=1$, we have $\operatorname{tr}(\tau \mid \delta(s \mathcal{L}))=P(a)$. It follows that $\operatorname{tr}(\tau \mid \delta(s \mathcal{F}))=M_{X}^{P}(\operatorname{cl}(\mathcal{F}))$ with $M_{X}^{P}$ as in Section 1; this holds for all generators $\tau$ of $G$, so $\delta(s \mathcal{F})=M_{X}^{P}(\operatorname{cl}(\mathcal{F}))$ in $R(k) \otimes K_{\sigma}(X)$. Putting $\mathcal{F}=\Omega_{X}$ then yields the result.

TheOREM 3.4. Assume that $X$ is smooth. For every $a \in K(X)$,

$$
S a=i_{*}\left(\theta^{n}(X)^{-1} \cdot \psi_{X}^{n}(a)\right)
$$

holds in $\mathbb{Z}\left[\frac{1}{n}\right] \otimes K_{\sigma}(X)$.

As in 3.2 and 3.3 , the right-hand side belongs to $\mathbb{Z}\left[\frac{1}{n}\right] \otimes K(X)$. Theorem 3.4 is immediate from 2.8, 3.2, and 3.3.

Proposition 3.5. Assume that $X$ is complete. For all $a \in \mathcal{G}(X)$,

holds in $K_{\sigma}(\operatorname{Spec}(k))$.

$$
\chi_{\sigma}(Y, S a)=\psi^{n} \chi(X, a)
$$


Once again, $\chi(X, a) \in K(\operatorname{Spec}(k))=\mathbb{Z}$ and so $\psi^{n} \chi(X, a)=\chi(X, a)$.

Proof. It suffices to check this for $a=\operatorname{cl}(\mathcal{F})$ where $\mathcal{F}$ is a coherent sheaf on $X$. The cohomologies of $S \mathcal{F}$ on $Y$ are computed by the Kunneth formula, and the off-diagonal terms can be dropped because they are induced from proper subgroups. The $G$-representation on $H^{p}(X, \mathcal{F}) \otimes H^{p}(X, \mathcal{F}) \otimes \cdots \otimes H^{p}(X, \mathcal{F}) \subset$ $H^{n p}(Y, S \mathcal{F})$ is the permutation representation tensored with $\varepsilon^{p}$, where $\varepsilon: G \rightarrow$ $\{ \pm 1\}$ is the sign of the permutation. By Lemma 3.2 applied to $\operatorname{Spec}(k)$, the image of the former in $K_{\sigma}(\operatorname{Spec}(k))$ is $\psi^{n}\left(\operatorname{cl} H^{p}(X, \mathcal{F})\right)=\operatorname{rk} H^{p}(X, \mathcal{F}) \in \mathbb{Z} \subset$ $K_{\sigma}(\operatorname{Spec}(k))$. Because the image of $\varepsilon \in K_{G}(\operatorname{Spec}(k)) \rightarrow K_{\sigma}(\operatorname{Spec}(k))$ is $(-1)^{n+1}$, it follows that

$$
\begin{aligned}
\chi_{\sigma}(Y, S a) & =\sum_{p}(-1)^{p n} \cdot(-1)^{p(n+1)} \cdot \operatorname{rk} H^{p}(X, \mathcal{F}) \\
& =\sum_{p}(-1)^{p} \operatorname{rk} H^{p}(X, \mathcal{F}) \\
& =\chi(X, \mathcal{F}) .
\end{aligned}
$$

THEOREM 3.6 (ARR). If $X$ is complete and smooth and if $a \in K(X)$, then $\chi(X, a)=\chi\left(X, \theta^{n}(X)^{-1} \cdot \psi_{X}^{n}(a)\right)$.

Proof. By Theorem 3.4,

$$
\chi_{\sigma}(Y, S a)=\chi_{\sigma}\left(X, \theta^{n}(X)^{-1} \cdot \psi_{X}^{n}(a)\right)=\chi\left(X, \theta^{n}(X)^{-1} \cdot \psi_{X}^{n}(a)\right) .
$$

But $\chi_{\sigma}(Y, S a)=\chi(X, \mathcal{F})$ from Proposition 3.5. This completes the proof of ARR.

\section{The Hirzebruch-Riemann-Roch Theorem}

The notation is as in Section 3; in addition, we assume that $k$ is algebraically closed. For HRR, we may assume this without any loss of generality.

Notation 4.1. $\quad X$ is smooth of dimension $d$. For every coherent sheaf $\mathcal{F}$ on $X$, we have $\operatorname{cl}(\mathcal{F}) \in \mathcal{G}(X)=K(X)$. We use $\mathcal{G}^{p}(X)$ to denote the subgroup of $\mathcal{G}(X)$ generated by $\operatorname{cl}(\mathcal{F})$ with $\operatorname{dim} \operatorname{supp}(\mathcal{F}) \leq d-p$. We put $K^{p}(X)=\{a \in \mathbb{Q} \otimes K(X)$ : $\left.\psi_{X}^{n}(a)=n^{p} a\right\}$.

Proposition 4.2 is proved in [GS]; a proof is included here for completeness.

Proposition 4.2. $\mathbb{Q} \otimes \mathcal{G}^{p}(X)=\bigoplus_{q \geq p} K^{q}(X)$.

Notation 4.3. Let $\pi: \mathbb{Q} \otimes K(X) \rightarrow K^{d}(X)$ denote the projection obtained from $p=0$ and $q=d$ in Proposition 4.2.

REMARK 4.4. Assume, in addition, that $X$ is complete. Then $a \mapsto \int_{X} \operatorname{ch}(a)$ is a homomorphism from $\mathcal{G}(X)$ to $(n !)^{-1} \mathbb{Z}$. Restrict this homomorphism to $\mathcal{G}^{d}(X)$. The kernel of $\chi(X, \cdot): \mathcal{G}^{d}(X) \rightarrow \mathbb{Z}$ is a divisible group because Jacobians of curves are divisible. It follows that there is $e(X) \in(n !)^{-1} \mathbb{Z}$ so that 


$$
\int_{X} \operatorname{ch}(a)=e(X) \chi(X, a)
$$

for all $a \in \mathcal{G}^{d}(X)$. If $f: X_{1} \rightarrow X_{2}$ is a surjective morphism (both smooth and complete of dimension $d$ ) then, by considering $f^{*} a$ for $a \in \mathcal{G}^{d}\left(X_{2}\right)$, we see that $e\left(X_{1}\right)=e\left(X_{2}\right)$. Checking that $e\left(\mathbb{P}^{d}\right)=1$, by Chow's lemma we see that $e(X)=$ 1 for all smooth complete $X$.

Proof of HRR. We proved ARR in Section 3; thus we may assume ARR' (see Section 1). For $a \in \mathbb{Q} \otimes K(X)$, we put $\chi^{\prime}(a)=\chi\left(X, \tilde{\operatorname{td}}(X)^{-1} a\right)$. By $\mathrm{ARR}^{\prime}$, we have

$$
\chi^{\prime}(a)=n^{-d} \cdot \chi^{\prime}\left(\psi_{X}^{n} a\right) \text { for all } a \in \mathbb{Q} \otimes K(X) .
$$

It follows that $\chi^{\prime}(a)=0$ for all $a \in K^{p}(X)$ and for all $p \neq d$. In other words, $\chi^{\prime}(a)=\chi^{\prime}(\pi a)$ with $\pi$ as in 4.3. Also, for $a \in \mathbb{Q} \otimes \mathcal{G}^{d}(X), a=\widetilde{\operatorname{td}}(X)^{-1} a$ because $P(1)=1$, where $P(t)=\log t /(t-1)$. Thus $\chi^{\prime}(a)=\chi(X, a)=\int_{X} \operatorname{ch}(a)$ from 4.4 if $a \in \mathbb{Q} \otimes \mathcal{G}^{d}(X)$. Finally, because $\operatorname{ch}\left(K^{p}(X)\right) \subset \mathbb{Q} \otimes A^{p}(X)$, we see that $\int_{X} \operatorname{ch}(a)=\int_{X} \operatorname{ch}(\pi a)$ for all $a \in \mathbb{Q} \otimes K(X)$. It follows that

$$
\chi\left(X, \tilde{\operatorname{td}}(X)^{-1} a\right)=\chi^{\prime}(a)=\chi^{\prime}(\pi a)=\int_{X} \operatorname{ch}(\pi a)=\int_{X} \operatorname{ch}(a),
$$

and replacing $a$ by $\widetilde{\operatorname{td}}(X) a$ yields the statement of HRR.

Proof of Proposition 4.2. For any closed subscheme $Z$ of $X$, we have $[Z]=$ $\operatorname{cl}\left(\mathcal{O}_{Z}\right) \in \mathcal{G}(X)=K(X)$. It is standard that $\mathcal{G}^{p}(X) / \mathcal{G}^{p+1}(X)$ is generated by $[Z]$ for $Z$ closed and irreducible of codimension $p$ in $X$. To prove 4.2 (by decreasing induction on $p$ ), it suffices to prove that $\psi_{X}^{n}[Z]-n^{p}[Z]$ is in $\mathcal{G}^{p+1}(X)$ for such $Z \subset X$.

Let $F$ be a finite locally free resolution of $\mathcal{O}_{Z}$. We put $D=F^{\otimes^{n}}$; thus, $D$ is a complex of $G$-sheaves on $X$. We see that

$$
\begin{aligned}
\psi_{X}^{n}[Z] & =\psi_{X}^{n}\left(\sum_{q}(-1)^{q} \operatorname{cl}\left(F_{q}\right)\right) \\
& =\sum_{q}(-1)^{q} \operatorname{cl}\left(F_{q}^{\otimes^{n}}\right) \text { in } \mathbb{Z}\left[\frac{1}{n}\right] \otimes \mathcal{G}_{\sigma}(X) \quad(\text { by Lemma 3.2) } \\
& =\sum_{q}(-1)^{q} \operatorname{cl}\left(D_{q}\right) \quad \text { (ignoring the off-diagonal terms as with 3.5) } \\
& =\sum_{q}(-1)^{q} \operatorname{cl}\left(H^{q}(D)\right) .
\end{aligned}
$$

This is an equality in $\mathbb{Z}\left[\frac{1}{n}\right] \otimes \mathcal{G}_{\sigma}(X)$. Let $\mathcal{I}$ be the sheaf of ideals in $\mathcal{O}_{X}$ that annihilates $\mathcal{O}_{Z}$. Then $\mathcal{I}$ annihilates the $G$-sheaf $H_{q}(D)$ and $\operatorname{tr}\left(\tau \mid H_{q}(D)\right)$ for any $\tau \in$ gen. If $m=\sum_{q}(-1)^{q} l\left(\operatorname{tr}\left(\tau \mid H_{q}(D)\right)\right.$, where $l$ denotes the length of the sheaf at the generic point of $Z$, then $\psi_{X}^{n}[Z]-m[Z] \in \mathcal{G}^{p+1}(X)$, so we have to prove $m=$ $n^{p}$. For this, we may replace $X$ by any Zariski-open subset whose intersection 
with $Z$ is nonempty. Thus we may assume that $Z$ is a local complete intersection in $X$. Let $j: Z \rightarrow X$ denote the inclusion and define $N \in K(Z)$ by $j_{*} N^{*}=$ $\mathcal{I} / \mathcal{I}^{2}$. With $s$ as in the proof of Lemma 3.3, we see that $H_{q}(D)=j_{*} \bigwedge^{q}\left(s N^{*}\right)$ as a $G$-sheaf, just by checking the action of transpositions in the permutation group. It follows, as in the proof of 3.3, that $\sum_{q}(-1)^{q} \operatorname{cl}\left(H_{q}(D)\right)=j_{*}\left(M_{Z}^{P}\left(N^{*}\right)\right)$ where $P(t)=\left(t^{n}-1\right) /(t-1)$, and the equality holds in $\mathbb{Z}\left[\frac{1}{n}\right] \otimes \mathcal{G}_{\sigma}(X)$. Because $P(1)=$ $n$ and rk $N^{*}=p$, it follows that $m=n^{p}$ as desired; this completes the proof of the proposition. We remark that this method of proof also yields ARR for closed immersions directly.

\section{References}

[AB] M. F. Atiyah and R. Bott, A Lefschetz fixed point formula for elliptic differential operators, Bull. Amer. Math. Soc. 72 (1966), 245-50.

[BS] A. Borel and J.-P. Serre, Le théorème de Riemann-Roch (d'après Grothendieck), Bull. Soc. Math. France 86 (1958), 97-136.

[Fa] G. Faltings, Lectures on the arithmetic Riemann-Roch theorem, Ann. of Math. Stud., 127, Princeton Univ. Press, Princeton, NJ, 1992.

[F] W. Fulton, Intersection theory, Ergeb. Math. Grenzgeb. (3) 2, Springer-Verlag, Berlin, 1984.

[FL] W. Fulton and S. Lang, Riemann-Roch algebra, Grundlehren Math. Wiss., 277, Springer-Verlag, Berlin, 1985.

[GS] H. Gillet and C. Soule, Intersection theory using Adams operations, Invent. Math. 90 (1987), 243-277.

[M] J. I. Manin, Lectures on the K-functor in algebraic geometry, Russian Math. Surveys 24 (1969), 1-89.

[P] V. K. Patodi, An analytic proof of Riemann-Roch-Hirzebruch theorem for Kaehler manifolds, J. Differential Geom. 5 (1971), 251-283.

[Q] D. Quillen, Higher algebraic K-theory. I., Lecture Notes in Math., 341, Springer-Verlag, Berlin, 1973.

[SGA] P. Berthelot et al., Theorie des intersections et theoreme de Riemann-Roch. Seminaire de geometrie algebrique du Bois-Marie 1966-67, Lecture Notes in Math., 225, Springer-Verlag, Berlin, 1971.

[TT1] D. Toledo and Y. L. Tong, A parametrix for $\bar{\partial}$ and Riemann-Roch in Cech theory, Topology 15 (1976), 273-301.

[TT2] - Duality and intersection theory in complex manifolds. II. The holomorphic Lefschetz formula, Ann. of Math. (2) 108 (1978), 519-538.

University of Chicago

5734 S. University Ave.

Chicago, IL 60637

nori@math.uchicago.edu 